\title{
On the qualitative behavior of the solutions to second-order neutral delay differential equations
}

\author{
Shyam Sundar Santra ${ }^{1}$, Hammad Alotaibi ${ }^{2}$ and Omar Bazighifan ${ }^{3,4^{*}}$ (D)
}

\section{"Correspondence:}

o.bazighifan@gmail.com

${ }^{3}$ Department of Mathematics,

Faculty of Science, Hadhramout

University, Hadhramout, 50512, Yemen

${ }^{4}$ Department of Mathematics, Faculty of Education, Seiyun University, Hadhramout, 50512, Yemen

Full list of author information is available at the end of the article

\begin{abstract}
Differential equations of second order appear in numerous applications such as fluid dynamics, electromagnetism, quantum mechanics, neural networks and the field of time symmetric electrodynamics. The aim of this work is to establish necessary and sufficient conditions for the oscillation of the solutions to a second-order neutral differential equation. First, we have taken a single delay and later the results are generalized for multiple delays. Some examples are given and open problems are presented.
\end{abstract}

Keywords: Oscillation; Non-oscillation; Delay; Neutral; Lebesgue's Dominated Convergence theorem; Necessary and sufficient conditions

\section{Introduction}

Consider the class of nonlinear neutral delay differential equations of the form

$$
\left(a\left(w^{\prime}\right)^{\mu}\right)^{\prime}(y)+c(y) g(u(\varsigma(y)))=0,
$$

where $w(y)=u(y)+b(y) u(\vartheta(y))$ and $\mu$ is the ratio of two odd positive integers. We assume the following conditions hold.

(A1) $a, c, \vartheta, \varsigma \in C\left(\mathbb{R}_{+}, \mathbb{R}_{+}\right)$such that $\vartheta(y) \leq y, \varsigma(y) \leq y$ for $y \geq y_{0}, \vartheta(y) \rightarrow \infty, \varsigma(y) \rightarrow \infty$ as $y \rightarrow \infty$.

(A2) $g \in C(\mathbb{R}, \mathbb{R})$ is non-decreasing and odd with $u g(u)>0$ for $u \neq 0$.

(A3) $a(y)>0$ and $\int_{0}^{\infty}(a(\eta))^{-1 / \mu} d \eta=\infty$. By letting $A(y)=\int_{0}^{y}(a(\eta))^{-1 / \mu} d \eta$, we have $\lim _{y \rightarrow \infty} A(y)=\infty$.

(A4) $b \in C\left(\mathbb{R}_{+}, \mathbb{R}_{-}\right)$with $-1+(2 / 3)^{1 / \mu} \leq-b_{0} \leq b(y) \leq 0$ for $y \in \mathbb{R}_{+}$.

(A5) $b \in C\left(\mathbb{R}_{+}, \mathbb{R}_{-}\right)$with $-1<-b_{0} \leq b(y) \leq 0$ for $y \in \mathbb{R}_{+}$.

In 1978, Brands [1] showed that the solutions to

$$
u^{\prime \prime}(y)+c(y) u(y-\varsigma(y))=0
$$

are oscillatory, if and only if, the solutions to $u^{\prime \prime}(y)+c(y) u(y)=0$ are oscillatory. Baculikova et al. [2] considered (1) and studied the oscillatory behavior of (1) for $g(u)=u$,

(c) The Author(s) 2020. This article is licensed under a Creative Commons Attribution 4.0 International License, which permits use sharing, adaptation, distribution and reproduction in any medium or format, as long as you give appropriate credit to the original author(s) and the source, provide a link to the Creative Commons licence, and indicate if changes were made. The images or other third party material in this article are included in the article's Creative Commons licence, unless indicated otherwise in a credit line to the material. If material is not included in the article's Creative Commons licence and your intended use is not permitted by statutory regulation or exceeds the permitted use, you will need to obtain permission directly from the copyright holder. To view a copy of this licence, visit http://creativecommons.org/licenses/by/4.0/. 
$0 \leq b(y) \leq b_{0}<\infty$ and (A3). They obtained sufficient conditions for the oscillation of the solutions of the linear counterpart of (1), using comparison techniques. Chatzarakis et al. [3] considered the equation

$$
\left(a\left(u^{\prime}\right)^{\mu_{2}}\right)^{\prime}(y)+c(y) u^{\mu_{2}}(\varsigma(y))=0 .
$$

Also, Chatzarakis et al. [4] studied (2) to obtain new oscillation criteria. Džurina [5] studied the linear counterpart of (1) when $0 \leq b(y) \leq b_{0}<\infty$ and (A3) and established sufficient conditions for the oscillation of the solutions of the linear counterpart of (1) by comparison techniques. Karpuz et al. [6] studied (1) for various ranges of the neutral coefficient $b$. Pinelas and Santra [7] studied necessary and sufficient conditions for the solutions of

$$
(u(y)+b(y) u(y-\vartheta))^{\prime}+\sum_{i=1}^{m} c_{j}(y) g\left(u\left(y-\varsigma_{j}\right)\right)=0
$$

Wong [8] obtained necessary and sufficient conditions for the oscillation of

$$
(u(y)+b u(y-\vartheta))^{\prime \prime}+c(y) g(y-\varsigma)=0,
$$

where the constant $b$ satisfies $-1<b<0$. Grace et al. [9] studied (1) and established sufficient conditions for $0 \leq b(y)<1$. For further work on this type of equations, we refer the reader to [10-36] and the references cited therein. We may note that most of the authors considered only sufficient conditions, and only a few considered necessary and sufficient conditions. Hence, the objective of this work is to establish both necessary and sufficient conditions for oscillation of (1) without using comparison techniques.

In Sect. 2 some preliminary results are presented, Sect. 3 deals with main results, Sect. 4 represents the conclusion and the final section includes open problems.

\section{Preliminary results}

In this section, two lemmas are presented which we need for our work in the sequel.

Lemma 2.1 Under the assumptions (A1)-(A3) and (A4) or (A5) and the solution $u$ of (1) is an eventually positive solution, we have

(i) $w(y)<0, w^{\prime}(y)>0$ and $\left(a\left(w^{\prime}\right)^{\mu}\right)^{\prime}(y)<0$;

(ii) $w(y)>0, w^{\prime}(y)>0$ and $\left(a\left(w^{\prime}\right)^{\mu}\right)^{\prime}(y)<0$, for sufficiently large $y$.

Proof Assume there exists a $y_{1} \geq y_{0}$ such that $u(y)>0, u(\vartheta(y))$, and $u(\varsigma(y))>0$ for $y \geq y_{1}$. From (1) and (A2), we have

$$
\left(a\left(w^{\prime}\right)^{\mu}\right)^{\prime}(y)=-c(y) g(u(\varsigma(y)))<0 \quad \text { for } y \geq y_{1},
$$

which implies that $\left(a\left(w^{\prime}\right)^{\mu}\right)(y)$ is non-increasing on $\left[y_{1}, \infty\right)$. We have $a(y)>0$, and thus either $w^{\prime}(y)<0$ or $w^{\prime}(y)>0$ for $y \geq y_{2}$, where $y_{2} \geq y_{1}$.

If $w^{\prime}(y)>0$ for $y \geq y_{2}$, then we have (i) and (ii). We prove now that $w^{\prime}(y)<0$ cannot occur. 
If $w^{\prime}(y)<0$ for $y \geq y_{2}$, then there exists $\kappa_{1}>0$ such that $\left(a\left(w^{\prime}\right)^{\mu}\right)(y) \leq-\kappa_{1}$ for $y \geq y_{2}$, which yields upon integration over $\left[y_{2}, y\right) \subset\left[y_{2}, \infty\right)$ after dividing through by $a$

$$
w(y) \leq w\left(y_{2}\right)-\kappa_{1}^{1 / \mu} \int_{y_{2}}^{y}(a(\eta))^{-1 / \mu} d \eta \quad \text { for } y \geq y_{2} .
$$

By virtue of condition (A3), $\lim _{t \rightarrow \infty} w(y)=-\infty$. We consider the following possibilities:

Let the solution $u$ be unbounded. There exists a sequence $\left\{y_{k}\right\}$ such that $\lim _{k \rightarrow \infty} y_{k}=\infty$ and $\lim _{k \rightarrow \infty} u\left(y_{k}\right)=\infty$, where $u\left(y_{k}\right)=\max \left\{u(\eta): y_{0} \leq \eta \leq y_{k}\right\}$. Since $\lim _{y \rightarrow \infty} \vartheta(y)=\infty$, $\vartheta\left(y_{k}\right)>y_{0}$ for all sufficiently large $k$. By $\vartheta(y) \leq y$,

$$
u\left(\vartheta\left(y_{k}\right)\right)=\max \left\{u(\eta): y_{0} \leq \eta \leq \vartheta\left(y_{k}\right)\right\} \leq \max \left\{u(\eta): y_{0} \leq \eta \leq y_{k}\right\}=u\left(y_{k}\right) .
$$

Therefore, for all large $k$,

$$
w\left(y_{k}\right)=u\left(y_{k}\right)+b\left(y_{k}\right) u\left(\vartheta\left(y_{k}\right)\right) \geq\left(1+b\left(y_{k}\right)\right) u\left(y_{k}\right)>0,
$$

which contradicts $\lim _{y \rightarrow \infty} w(y)=-\infty$.

Let the solution $u$ be bounded, then $w$ is bounded, from which one concludes $\lim _{y \rightarrow \infty} w(y)=-\infty$, a contradiction. Hence, $w$ satisfies one of the cases (i) or (ii). This completes the proof.

Lemma 2.2 Under the assumptions (A1)-(A3), (A4) or (A5), (i) and $u$ is an eventually positive solution of (1), we have $\lim _{y \rightarrow \infty} u(y)=0$.

Proof Assume that there exists a $y_{1} \geq y_{0}$ such that $u(y)>0, u(\vartheta(y))$, and $u(\varsigma(y))>0$ for $y \geq y_{1}$. Then Lemma 2.1 holds and $w$ satisfies one of the cases (i) or (ii) for $y_{2} \geq y_{1}$, where $y \geq y_{2}$. Let $w$ satisfy (i) for $y \geq y_{2}$. Therefore,

$$
\begin{aligned}
0 & \geq \lim _{y \rightarrow \infty} w(y)=\limsup _{y \rightarrow \infty} w(y) \geq \limsup _{y \rightarrow \infty}\left(u(y)-b_{0} u(\vartheta(y))\right) \\
& \geq \limsup _{y \rightarrow \infty} u(y)+\liminf _{t \rightarrow \infty}\left(-b_{0} u(\vartheta(y))\right)=\left(1-b_{0}\right) \limsup _{y \rightarrow \infty} u(y),
\end{aligned}
$$

which implies that $\limsup _{y \rightarrow \infty} u(y)=0$ and hence $\lim _{y \rightarrow \infty} u(y)=0$.

Remark 1 In view of (ii) of Lemma 2.1, it is obvious that $\lim _{y \rightarrow \infty} w(y)>0$, i.e., there exists $\kappa_{1}>0$ such that $w(y) \geq \kappa_{1}$ for all large $y$.

\section{Main results}

In this section, we establish the necessary and sufficient conditions for the oscillation of the solution of (1) by considering the two cases when $g(v) / \nu^{\mu_{1}}$ is non-increasing and $g(v) / v^{\mu_{1}}$ is non-decreasing.

\subsection{The case when $g(v) / v^{\mu_{1}}$ is non-increasing}

Suppose that there exists $\mu_{1}$ such that $0<\mu_{1}<\mu$ and

$$
\frac{g(v)}{v^{\mu_{1}}} \geq \frac{g(u)}{u^{\mu_{1}}} \quad \text { for } 0<v \leq u .
$$


For example the function $g(u)=|u|^{\mu_{2}} \operatorname{sgn}(u)$ with $0<\mu_{2}<\mu_{1}<\mu$ satisfying (5).

Theorem 3.1 Assume that (A1)-(A4) and (5) hold. Then each unbounded solution of (1) is oscillatory if and only if

$$
\int_{Y}^{\infty} c(\eta) g\left(\kappa^{1 / \mu} A(\varsigma(\eta))\right) d \eta=+\infty \quad \forall Y>0 \text { and } \kappa>0
$$

Proof On the contrary, we assume that there exists a nonoscillatory unbounded solution $u(y)$ of (1). Suppose that the solution $u(y)$ is eventually positive. Then there exists $y_{1} \geq y_{0}$ such that $u(y)>0, u(y)>0, u(\vartheta(y))>0$ and $u(\varsigma(y))>0$ for $y \geq y_{1}$. Proceeding as in the proof of Lemma 2.1, we see that $\left(a\left(w^{\prime}\right)^{\mu}\right)(y)$ is non-increasing, and $w$ satisfies one of the cases (i) or (ii) on $\left[y_{2}, \infty\right)$, where $y_{2} \geq y_{1}$. Then we have the following two possible cases.

Case 1. Let $w$ satisfy (i) for $y \geq y_{2}$. As $u$ is the unbounded solution, there exists $y \geq y_{2}$ such that $u(y)=\max \left\{u(s): y_{2} \leq s \leq T\right\}$. Since $w(y)=u(y)+b(y) u(\vartheta(y))$, we have $u(y) \leq$ $w(y)+\left\{1-(2 / 3)^{1 / \mu}\right\} u(\vartheta(y))<u(y)$, which leads a contradiction.

Case 2. Let $w$ satisfy (ii) for $y \geq y_{2}$. Note that $\lim _{y \rightarrow \infty}\left(a\left(w^{\prime}\right)^{\mu}\right)(y)$ exists. Using $w(y) \leq u(y)$ in (1) and integrating the new inequality from $y$ to $+\infty$, we obtain

$$
\int_{y}^{\infty} c(\eta) g(w(\varsigma(\eta))) d \eta \leq\left(a\left(w^{\prime}\right)^{\mu}\right)(y)
$$

That is,

$$
w^{\prime}(y) \geq\left[\frac{1}{a(y)} \int_{y}^{\infty} c(\eta) g(w(\varsigma(\eta))) d \eta\right]^{1 / \mu}
$$

for $y \geq y_{3}$. Let $y_{4}>y_{3}$ be a point such that

$$
A(y)-A\left(y_{3}\right) \geq \frac{1}{2} A(y), \quad y \geq y_{4} .
$$

Then integrating (7) from $y_{3}$ to $y$, we get

$$
\begin{aligned}
w(y)-w\left(y_{3}\right) & \geq \int_{y_{3}}^{y}\left[\frac{1}{a(\eta)} \int_{\eta}^{\infty} c(\zeta) g(w(\varsigma(\zeta))) d \zeta\right]^{1 / \mu} d \eta \\
& \geq \int_{y_{3}}^{y}\left[\frac{1}{a(\eta)} \int_{y}^{\infty} c(\zeta) g(w(\varsigma(\zeta))) d \zeta\right]^{1 / \mu} d \eta
\end{aligned}
$$

i.e.,

$$
\begin{aligned}
w(y) & \geq\left(A(y)-A\left(y_{3}\right)\right)\left[\int_{y}^{\infty} c(\zeta) g(w(\varsigma(\zeta))) d \zeta\right]^{1 / \mu} \\
& \geq \frac{1}{2} A(y)\left[\int_{y}^{\infty} c(\zeta) g(w(\varsigma(\zeta))) d \zeta\right]^{1 / \mu} .
\end{aligned}
$$

Since $\left(a\left(w^{\prime}\right)^{\mu}\right)(y)$ is non-increasing on $\left[y_{4}, \infty\right)$, there exist $\kappa>0$ and $y_{5}>y_{4}$ such that $\left(a\left(w^{\prime}\right)^{\mu}\right)(y) \leq \kappa$ for $y \geq y_{5}$. Integrating the inequality $w^{\prime}(y) \leq(\kappa / a(y))^{1 / \mu}$, we have

$$
w(y) \leq w\left(y_{5}\right)+\kappa^{1 / \mu}\left(A(y)-A\left(y_{5}\right)\right) .
$$


Since $\lim _{t \rightarrow \infty} A(y)=\infty$, the last inequality becomes

$$
w(y) \leq \kappa^{1 / \mu} A(y) \quad \text { for } y \geq y_{5}
$$

On the other hand, (5) implies that

$$
g(w(\varsigma(\zeta)))=\frac{g(w(\varsigma(\zeta)))}{w^{\mu_{1}}(\varsigma(\zeta))} w^{\mu_{1}}(\varsigma(\zeta)) \geq \frac{g\left(\kappa^{1 / \mu} A(\varsigma(\zeta))\right)}{\left(\kappa^{1 / \mu} A(\varsigma(\zeta))\right)^{\mu_{1}}} w^{\mu_{1}}(\varsigma(\zeta))
$$

Consequently, (8) becomes

$$
w(y) \geq \frac{A(y)}{2}\left[\int_{y}^{\infty} \frac{c(\zeta) g\left(\kappa^{1 / \mu} A(\varsigma(\zeta))\right) w^{\mu_{1}}(\varsigma(\zeta))}{\left(\kappa^{1 / \mu} A(\varsigma(\zeta))\right)^{\mu_{1}}} d \zeta\right]^{1 / \mu}
$$

If we define

$$
\Upsilon(y)=\int_{y}^{\infty} \frac{c(\zeta) g\left(\kappa^{1 / \mu} A(\varsigma(\zeta))\right) w^{\mu_{1}}(\varsigma(\zeta))}{\left(\kappa^{1 / \mu} A(\varsigma(\zeta))\right)^{\mu_{1}}} d \zeta
$$

then $w^{\mu_{1}} /\left(\kappa^{1 / \mu} A\right)^{\mu_{1}} \geq \Upsilon^{\mu_{1} / \mu} /\left(2 \kappa^{1 / \mu}\right)^{\mu_{1}}$. Taking the derivative of $\Upsilon$ we get

$$
\Upsilon^{\prime}(y) \leq-\frac{g\left(\kappa^{1 / \mu} A(\varsigma(y))\right) c(y) w^{\mu_{1}}(\varsigma(y))}{\left(\kappa^{1 / \mu} A(\varsigma(y))\right)^{\mu_{1}}} \leq-\frac{c(y) g\left(\kappa^{1 / \mu} A(\varsigma(y))\right)}{\left(2 \kappa^{1 / \mu}\right)^{\mu_{1}}} \Upsilon^{\mu_{1} / \mu}(\varsigma(y)) \leq 0 .
$$

Therefore, $\Upsilon(y)$ is non-increasing on $\left[y_{5}, \infty\right)$ so $\Upsilon^{\mu_{1} / \mu}(\varsigma(y)) / \Upsilon^{\mu_{1} / \mu}(y) \geq 1$, and

$$
\begin{aligned}
\left(\Upsilon^{1-\mu_{1} / \mu}(y)\right)^{\prime} & \leq-\left(1-\mu_{1} / \mu\right) \Upsilon^{-\mu_{1} / \mu}(y) \frac{c(y) g\left(\kappa^{1 / \mu} A(\varsigma(y))\right)}{\left(2 \kappa^{1 / \mu}\right)^{\mu_{1}}} \Upsilon^{\mu_{1} / \mu}(\varsigma(y)) \\
& \leq-\left(1-\mu_{1} / \mu\right) \frac{c(y) g\left(\kappa^{1 / \mu} A(\varsigma(y))\right)}{\left(2 \kappa^{1 / \mu}\right)^{\mu_{1}}} .
\end{aligned}
$$

We have $\mu_{1} / \mu<1$ and $\Upsilon(y)$ is positive and non-increasing. Integrating the last inequality, from $y_{5}$ to $y$, we have

$$
\frac{\left(1-\mu_{1} / \mu\right)}{\left(2 \kappa^{1 / \mu}\right)^{\mu_{1}}} \int_{t^{5}}^{y} c(\eta) g\left(\kappa^{1 / \mu} A(\varsigma(\eta))\right) d \eta \leq-\left[\Upsilon^{1-\mu_{1} / \mu}(\eta)\right]_{y_{5}}^{y}<\Upsilon^{1-\mu_{1} / \mu}\left(y_{5}\right)<\infty
$$

which contradicts (6).

If $u(y)<0$ for $y \geq y_{1}$, then we set $y(y):=-u(y)$ for $y \geq y_{1}$ in (1). Using (A2), we find

$$
\left(a(y)\left(\bar{w}^{\prime}(y)\right)^{\mu}\right)+c(y) \bar{g}(y(\varsigma(y)))=0 \quad \text { for } y \geq y_{1},
$$

where $\bar{w}(y)=y(y)+b(y) y(\vartheta(y))$ and $\bar{g}(u):=-g(-u)$ for $u \in \mathbb{R}$. Clearly, $\bar{g}$ satisfies (A2). Then, proceeding as above, we can find the same contradiction.

To prove the condition (6) is necessary, assume that (6) does not hold; so for some $\kappa>0$ and $y \geq y_{0}$ we have

$$
\int_{Y}^{\infty} c(\eta) g\left(\kappa^{1 / \mu} A(\varsigma(\eta))\right) d \eta \leq \frac{\kappa}{3}
$$


We set

$$
\begin{aligned}
S= & \left\{u: u \in C\left(\left[y_{0}, \infty\right), \mathbb{R}\right), u(y)=0 \text { for } y \in\left[y_{0}, Y\right]\right. \text { and } \\
& \left.\left(\frac{\kappa}{3}\right)^{1 / \mu}[A(y)-A(Y)] \leq u(y) \leq \kappa^{1 / \mu}[A(y)-A(Y)] \text { for } y \geq y_{0}\right\} .
\end{aligned}
$$

We define the operator $\Omega: S \rightarrow C\left(\left[y_{0},+\infty\right), \mathbb{R}\right)$ by

$$
(\Omega u)(y)= \begin{cases}0, & y \in\left[y_{0}, Y\right], \\ -b(y) u(\vartheta(y))+\int_{Y}^{y}\left[\frac{1}{a(\eta)}\left[\frac{\kappa}{3}+\int_{\eta}^{\infty} c(\zeta) g(u(\varsigma(\zeta))) d \zeta\right]\right]^{1 / \mu} d \eta, & y \geq Y .\end{cases}
$$

For every $u \in S$ and $y \geq Y$, we have

$$
\begin{aligned}
(\Omega u)(y) & \geq \int_{Y}^{y}\left[\frac{1}{a(\eta)}\left[\frac{\kappa}{3}+\int_{\eta}^{\infty} c(\zeta) g(u(\varsigma(\zeta))) d \zeta\right]\right]^{1 / \mu} d \eta \\
& \geq \int_{Y}^{y}\left[\frac{1}{a(\eta)} \frac{\kappa}{3}\right]^{1 / \mu} d \eta=\left(\frac{\kappa}{3}\right)^{1 / \mu}[A(y)-A(Y)] .
\end{aligned}
$$

For every $u \in S$ and $y \geq Y$, we have $u(y) \leq \kappa^{1 / \mu} A(y)$ and $g(u(y)) \leq g\left(\kappa^{1 / \mu} A(y)\right)$. Then

$$
\begin{aligned}
(\Omega u)(y) & \leq-b(y) u(\vartheta(y))+\int_{T}^{y}\left[\frac{1}{a(\eta)}\left(\frac{\kappa}{3}+\frac{\kappa}{3}\right)\right]^{1 / \mu} d \eta \\
& \leq b_{0} \kappa^{1 / \mu}[A(\vartheta(y))-A(Y)]+(2 \kappa / 3)^{1 / \mu}[A(y)-A(Y)] \\
& \leq b_{0} \kappa^{1 / \mu}[A(y)-A(Y)]+(2 \kappa / 3)^{1 / \mu}[A(y)-A(Y)] \\
& =\left(b_{0}+(2 / 3)^{1 / \mu}\right) \kappa^{1 / \mu}[A(y)-A(Y)] \leq \kappa^{1 / \mu}[A(y)-A(Y)],
\end{aligned}
$$

which implies that $(\Omega u)(y) \in S$. Let us define now a sequence of continuous function $v_{n}$ : $\left[y_{0},+\infty\right) \rightarrow \mathbb{R}$ by the recursive formula

$$
\begin{aligned}
& u_{0}(y)= \begin{cases}0, & y \in\left[y_{0}, Y\right], \\
\frac{\kappa}{3}[A(y)-A(Y)], & y \geq Y,\end{cases} \\
& u_{n}(y)=\left(\Omega u_{n-1}\right)(y), \quad n \geq 1 .
\end{aligned}
$$

Inductively, it is easy to verify that, for $n>1$,

$$
\left(\frac{\kappa}{3}\right)^{1 / \mu}[A(y)-A(Y)] \leq u_{n-1}(y) \leq u_{n}(y) \leq \kappa^{1 / \mu}[A(y)-A(Y)]
$$

Therefore the point-wise limit of the sequence exists. Let $\lim _{y \rightarrow \infty} u_{n}(y)=v(y)$ for $y \geq y_{0}$. By Lebesgue's dominated convergence theorem, $u \in S$ and $(\Omega u)(y)=u(y)$, where $u(y)$ is a solution of $(1)$ on $[Y, \infty)$ such that $u(y)>0$. Hence, (6) is necessary. This completes the proof. 
Example 3.2 Consider the delay differential equation

$$
\left(e^{-y}\left(\left(u(y)-e^{-y} u(y-1)\right)^{\prime}\right)^{3 / 5}\right)^{\prime}+y(u(y-2))^{1 / 3}=0, \quad y \geq 0 .
$$

Here $\mu=3 / 5, a(y)=e^{-y},-1<b(y)=-e^{-y} \leq 0, \vartheta(y)=y-1, \varsigma(y)=y-2, A(y)=\int_{0}^{y} e^{55 / 3} d s=$ $\frac{3}{5}\left(e^{5 y / 3}-1\right), g(v)=v^{1 / 3}$. For $\mu_{1}=1 / 2$, we have a decreasing function $g(v) / v^{\mu_{1}}=v^{-1 / 6}$. Now

$$
\int_{0}^{\infty} c(\eta) g\left(\kappa^{1 / \mu} A(\varsigma(\eta))\right) d \eta=\int_{0}^{\infty} \eta\left(\kappa^{5 / 3} \frac{3}{5}\left(e^{5(\eta-2 / 3}-1\right)\right)^{1 / 3} d \eta=\infty \quad \forall \kappa>0
$$

So, all the conditions of Theorem 3.1 hold, and therefore every unbounded solution of (9) is oscillatory.

Theorem 3.3 Let assumptions (A1)-(A4) hold. Then each unbounded solution of (1) oscillates if and only if (6) holds for every $\kappa>0$.

Proof To prove sufficiency by contradiction, assume that the solution $u$ of (1) is eventually positive and unbounded. So, there exists $y_{1} \geq y_{0}$ such that $u(y)>0, u(\vartheta(y))>0$ and $u(s(y))>0$ for $y \geq y_{1}$. Proceeding as in the proof of Lemma 2.1, $\left(a\left(w^{\prime}\right)^{\mu}\right)(y)$ is nonincreasing, $w$ satisfies one of the cases (i) or (ii) on $\left[y_{2}, \infty\right.$ ), where $y_{2} \geq y_{1}$. We have the following two possible cases.

Case 1. Let $w$ satisfy (i) for $y \geq y_{2}$. This case is similar to the proof of Theorem 3.1.

Case 2. Let $w$ satisfy (ii) for $y \geq y_{2}$. Since $w(y)$ is unbounded and monotonically increasing, it follows that

$$
\lim _{y \rightarrow \infty} \frac{w^{\mu}(y)}{A^{\mu}(y)}=\lim _{y \rightarrow \infty} \frac{\left(w^{\prime}(y)\right)^{\mu}}{\left(A^{\prime}(y)\right)^{\mu}}=\lim _{y \rightarrow \infty}\left(a\left(w^{\prime}\right)^{\mu}\right)(y)=c<\infty .
$$

If $c=0$, then $\lim _{t \rightarrow \infty} A(y)=+\infty$ implies that $\lim _{t \rightarrow \infty} w(y)<+\infty$, which is invalid $(\because w(y)$ is unbounded). Hence $c \neq 0$. Therefore, there exist a constant $\kappa>0$ and a $y_{2}>y_{1}$ such that $w(y) \geq \kappa^{1 / \mu} A(y)$ for $y \geq y_{2}$. Consequently, $u(y) \geq w(y) \geq \kappa^{1 / \mu} A(y)$ for $y \geq y_{2}$. Using $u(y) \geq \kappa^{1 / \mu} A(y)$ in (1) and then integrating the final inequality from $y_{2}$ to $+\infty$, we obtain a contradiction to (6) for every $\kappa>0$.

By using the same transformation as in the proof of Theorem 3.1 we can get a contradiction for an eventually negative unbounded solution, so we omit it here.

One can prove the necessary part by following the proof of Theorem 3.1. So we omit it here. The proof of the theorem is complete.

Theorem 3.4 Assume that (A1)-(A4) and (5) hold. Then each solution of (1) is oscillatory or $\lim _{y \rightarrow \infty} u(y)=0$ if and only if (6) holds for every $\kappa>0$.

Proof On the contrary, we assume that the solution $u$ of (1) is eventually positive. Then there exists $y_{1} \geq y_{0}$ such that $u(y)>0, u(\vartheta(y))>0$ and $u(\varsigma(y))>0$ for $y \geq y_{1}$. Proceeding as in the proof of Lemma 2.1, we see $\left(a\left(w^{\prime}\right)^{\mu}\right)(y)$ is non-increasing, and $w$ satisfies one of the cases (i) or (ii) on $\left[y_{2}, \infty\right)$, where $y_{2} \geq y_{1}$. Thus, we have the following two possible cases.

Case 1. Let $w$ satisfy (i) for $y \geq y_{2}$. Then, by Lemma 2.2, we have $\lim _{y \rightarrow \infty} u(y)=0$.

Case 2. Let $w$ satisfy (ii) for $y \geq y_{2}$. The case follows from the proof of Theorem 3.1.

The necessary part is similar to Theorem 3.1. The proof of the theorem is complete. 


\subsection{The case when $g(u) / u^{\mu_{1}}$ is non-decreasing}

Suppose that there exists $\mu_{1}>\mu$ such that

$$
\frac{g(v)}{v^{\mu_{1}}} \leq \frac{g(u)}{u^{\mu_{1}}} \quad \text { for } 0<v \leq u .
$$

For example we might consider the function $g(u)=|u|^{\mu_{2}} \operatorname{sgn}(u)$ with $\mu<\mu_{1}<\mu_{2}$ satisfying (10).

Theorem 3.5 Assume that (A1)-(A3), (A5), (10), $\varsigma^{\prime}(y) \geq 1$ hold. Then each solution of (1) oscillates or $\lim _{y \rightarrow \infty} u(y)=0$ if and only if

$$
\int_{Y}^{\infty}\left[\frac{1}{a(\zeta)}\left[\int_{\zeta}^{\infty} c(\eta) d \eta\right]\right]^{1 / \mu} d \zeta=+\infty \quad \forall y>0
$$

Proof Proceeding in the proof of Theorem 3.4, we can conclude that $\lim _{y \rightarrow \infty} u(y)=0$ when $z$ satisfies (i). Let us consider Case 2, for $y \geq y_{2}$. By Remark 1, there exist a constant $\kappa>0$ and $y_{2}>y_{1}$ such that $z(\varsigma(y)) \geq \kappa$ for $y \geq y_{2}$. Consequently,

$$
g(w(\varsigma(y)))=\frac{g(w(\varsigma(y)))}{w^{\mu_{1}}(\varsigma(y))} w^{\mu_{1}}(\varsigma(y)) \geq \frac{g(\kappa)}{\kappa^{\mu_{1}}} w^{\mu_{1}}(\varsigma(y))
$$

for $y \geq y_{2}$. Using $w(y) \leq u(x)$ and (12) in (1), and then integrating the final inequality we have

$$
\lim _{A \rightarrow \infty}\left[\left(a\left(w^{\prime}\right)^{\prime}\right)(\eta)\right]_{y}^{A}+\frac{g(\kappa)}{\kappa^{\mu_{1}}} \int_{y}^{\infty} c(\zeta) w^{\mu_{1}}(\varsigma(\zeta)) d \zeta \leq 0 .
$$

Since $\left(a\left(w^{\prime}\right)^{\prime}\right)(y)$ is non-increasing and positive, we have

$$
\frac{g(\kappa)}{\kappa^{\mu_{1}}} \int_{y}^{\infty} c(\eta) w^{\mu_{1}}(\varsigma(\eta)) d \eta \leq\left(a\left(w^{\prime}\right)^{\mu}\right)(y) \leq\left(a\left(w^{\prime}\right)^{\mu}\right)(\varsigma(y)) \leq a(y)\left(\left(w^{\prime}\right)^{\mu}\right)(\varsigma(y))
$$

for all $y \geq y_{2}$. Therefore,

$$
\left(\frac{g(\kappa)}{\kappa^{\mu_{1}}}\right)^{1 / \mu}\left[\frac{1}{a(y)}\left[\int_{y}^{\infty} c(\zeta) w^{\mu_{1}}(\varsigma(\zeta)) d \zeta\right]\right]^{1 / \mu} \leq w^{\prime}(\varsigma(y))
$$

implies that

$$
\left(\frac{g(\kappa)}{\kappa^{\mu_{1}}}\right)^{1 / \mu}\left[\frac{1}{a(y)}\left[\int_{y}^{\infty} c(\zeta) d \zeta\right]\right]^{1 / \mu} \leq \frac{w^{\prime}(\varsigma(y))}{w^{\mu_{1} / \mu}(\varsigma(y))} \leq \frac{w^{\prime}(\varsigma(y)) \varsigma^{\prime}(y)}{w^{\mu_{1} / \mu}(\varsigma(y))} .
$$

Integrating the final inequality from $y_{2}$ to $+\infty$, we have

$$
\begin{aligned}
\left(\frac{g(\kappa)}{\kappa^{\mu_{1}}}\right)^{1 / \mu} \int_{y_{2}}^{\infty}\left[\frac{1}{a(\zeta)}\left[\int_{\zeta}^{\infty} c(\eta) d \eta\right]\right]^{1 / \mu} d \zeta & <\int_{y_{2}}^{\infty} \frac{w^{\prime}(\varsigma(\eta)) \varsigma^{\prime}(\eta)}{w^{\mu_{1} / \mu}(\varsigma(\eta))} d \eta \\
& \leq \frac{w^{1-\mu_{1} / \mu}\left(\varsigma\left(y_{2}\right)\right)}{\mu_{1} / \mu-1}<\infty
\end{aligned}
$$

which contradicts (11). 
Next, we show that (11) is necessary. Assume that (11) does not hold and let there exist $y \geq y_{0}$ such that

$$
\int_{Y}^{y}\left[\frac{1}{a(\zeta)}\left[\int_{\zeta}^{\infty} c(\eta) d \eta\right]\right]^{1 / \mu} d \zeta \leq \frac{\left(1-b_{0}\right)(g(1))^{-1 / \mu}}{5}
$$

where $\kappa>0$ is a constant. We set

$$
S=\left\{u \in C\left(\left[y_{0}, \infty\right), \mathbb{R}\right): u(y)=\frac{1-b_{0}}{5}, y \in\left[y_{0}, Y\right] \frac{1-b_{0}}{5} \leq u(y) \leq 1 \text { for } y \geq Y\right\}
$$

We define the operator $\Omega: S \rightarrow C\left(\left[y_{0}, \infty\right), \mathbb{R}\right)$ by

$$
(\Omega u)(y)= \begin{cases}\frac{1-b_{0}}{5}, & y \in\left[y_{0}, Y\right] \\ -b(y) u(\vartheta(y))+\frac{1-b_{0}}{5}+\int_{T}^{y}\left[\frac{1}{a(\eta)}\left[\int_{\eta}^{\infty} c(\zeta) g(u(\varsigma(\zeta))) d \zeta\right]\right]^{1 / \mu} d \eta, & y \geq T .\end{cases}
$$

For every $u \in S$ and $y \geq Y,(\Omega u)(y) \geq \frac{1-b_{0}}{5}$ and

$$
\begin{aligned}
(\Omega u)(y) & \leq b_{0}+\frac{1-b_{0}}{5}+(g(1))^{1 / \mu} \int_{Y}^{y}\left[\frac{1}{a(\eta)}\left[\int_{\eta}^{\infty} c(\zeta) d \zeta\right]\right]^{1 / \mu} d \eta \\
& \leq b_{0}+\frac{1-b_{0}}{5}+\frac{1-b_{0}}{5}=\frac{3 b_{0}+2}{5}<1
\end{aligned}
$$

which implies that $\Omega u \in S$. The remaining proof follows from Theorem 3.1. This completes the proof.

Example 3.6 Consider the differential equation

$$
\left(\left(\left(u(y)-e^{-y} u(\vartheta(y))\right)^{\prime}\right)^{1 / 5}\right)^{\prime}+(y+1)(u(y-2))^{\frac{7}{3}}=0, \quad y \geq 0
$$

Here $\mu=1 / 5, a(y)=1, \varsigma(y)=y-2, g(v)=v^{\frac{7}{3}}$. For $\mu_{1}=4 / 3$, we have $g(v) / v^{\mu_{1}}=v$, which is an increasing function. To check (11) we have

$$
\int_{2}^{\infty}\left[\int_{\zeta}^{\infty}(\eta+1) d \eta\right]^{5} d \zeta=\infty
$$

So, all conditions of Theorem 3.5 hold, and therefore each solution of (13) oscillates or converges to zero.

\section{Conclusion}

It is worth noting that we have established the necessary and sufficient conditions when $-1<b(y) \leq 0$. These conditions do not hold in all ranges of $b(y)$.

Remark 2 Theorems 3.1-3.5 also hold for the following equation:

$$
\left(a(y)\left((u(y)+b(y) u(\vartheta(y)))^{\prime}\right)^{\mu}\right)^{\prime}+\sum_{i=1}^{m} c_{j}(y) g_{j}\left(u\left(\varsigma_{j}(y)\right)\right)=0
$$


where $b, a, c_{j}, g_{j}, \varsigma_{j}(j=1,2, \ldots, m)$ satisfy assumptions (A1)-(A5). In order to extend Theorems 3.1-3.5, we can find an index $i$ so that $c_{j}, g_{j}, \varsigma_{j}$ satisfies (6) and (11).

Example 4.1 Consider the neutral differential equation

$$
\left(e^{-y}\left(\left(u(y)-e^{-y} u(\vartheta(y))\right)^{\prime}\right)^{3 / 5}\right)^{\prime}+\frac{1}{y+1}(u(y-2))^{1 / 3}+\frac{1}{y+2}(u(y-1))^{1 / 5}=0, \quad y \geq 0 .
$$

Here $\mu=3 / 5, a(y)=e^{-y}, b(y)=-e^{-y}, \varsigma_{1}(y)=u-2, \varsigma_{2}(y)=u-1, A(y)=\int_{0}^{y} e^{5 s / 3} d s=\frac{3}{5}\left(e^{5 y / 3}-\right.$ 1), $g_{1}(v)=v^{1 / 3}$ and $g_{2}(v)=v^{1 / 5}$. For $\mu_{1}=1 / 2$, we have decreasing functions $g_{1}(v) / v^{\mu_{1}}=v^{-1 / 6}$ and $g_{2}(v) / v^{\mu_{1}}=v^{-3 / 10}$. Now,

$$
\begin{aligned}
\int_{0}^{\infty} & \sum_{i=1}^{m} c_{j}(\eta) g_{j}\left(\kappa^{1 / \mu} A\left(s_{j}(\eta)\right)\right) d \eta \\
& \geq \int_{0}^{\infty} g_{1}(\eta) f_{1}\left(\kappa^{1 / \mu} A\left(s_{1}(\eta)\right)\right) d \eta \\
& =\int_{0}^{\infty} \frac{1}{\eta+1}\left(\kappa^{5 / 3} \frac{3}{5}\left(e^{5(\eta-2) / 3}-1\right)\right)^{1 / 3} d \eta=\infty \quad \forall \kappa>0 .
\end{aligned}
$$

So, all the conditions of Theorem 3.1 hold, and therefore every unbounded solution of (14) is oscillatory.

Example 4.2 Consider the differential equation

$$
\left(\left(\left(u(y)-e^{-y} u(\vartheta(y))\right)^{\prime}\right)^{5 / 7}\right)^{\prime}+t(u(y-2))^{5 / 3}+(y+1)(u(y-1))^{3}=0, \quad y \geq 0 .
$$

Here $\mu=5 / 7, a(y)=1, \varsigma_{1}(y)=y-2, \varsigma_{2}(y)=y-1, g_{1}(v)=v^{5 / 3}$ and $g_{2}(v)=v^{3}$. For $\mu_{1}=4 / 3$, we have decreasing functions $g_{1}(v) / v^{\mu_{1}}=v^{1 / 3}$ and $g_{2}(v) / v^{\mu_{1}}=v^{5 / 3}$. Clearly, all the conditions of Theorem 3.5 hold. Thus, each solution of (15) oscillates or $\lim _{y \rightarrow \infty} u(y)=0$.

Remark 3 Examples 4.1 and 4.2 prove the feasibility and effectiveness of Remark 2.

\section{Open problem}

This work leads to some open problems:

1. Can we find necessary and sufficient conditions for the oscillation of solutions to second-order differential equation (1) for the other ranges of the neutral coefficient $b$ ?

2. Is it possible to generalize this work to fractional order?

\section{Acknowledgements}

The authors are thankful to the the editors and and the referees for their valuable suggestions and comments, which improved the content of this paper.

\section{Funding}

The authors received no direct funding for this work.

\section{Availability of data and materials}

Not applicable. 
Authors' contributions

The authors declare that they read and approved the final manuscript.

\section{Authors' information}

Not applicable.

\section{Author details}

'Department of Mathematics, JIS College of Engineering, Kalyani, 741235, India. ${ }^{2}$ Department of Mathematics, Faculty of Science, Taif University, Taif, 21944, Saudi Arabia. ${ }^{3}$ Department of Mathematics, Faculty of Science, Hadhramout University, Hadhramout, 50512, Yemen. ${ }^{4}$ Department of Mathematics, Faculty of Education, Seiyun University, Hadhramout, 50512, Yemen.

\section{Publisher's Note}

Springer Nature remains neutral with regard to jurisdictional claims in published maps and institutional affiliations.

Received: 21 July 2020 Accepted: 26 November 2020 Published online: 07 December 2020

\section{References}

1. Brands, J.J.M.S.: Oscillation theorems for second-order functional-differential equations. J. Math. Anal. Appl. 63(1), 54-64 (1978)

2. Baculikova, B., Dzurina, J.: Oscillation theorems for second order neutral differential equations. Comput. Math. Appl. 61, 94-99 (2011)

3. Chatzarakis, G.E., Dzurina, J., Jadlovska, I.: New oscillation criteria for second-order half-linear advanced differential equations. Appl. Math. Comput. 347, 404-416 (2019)

4. Chatzarakis, G.E., Jadlovska, I.: Improved oscillation results for second-order half-linear delay differential equations. Hacet. J. Math. Stat. 48(1), 170-179 (2019)

5. Džurina, J.: Oscillation theorems for second order advanced neutral differential equations. Tatra Mt. Math. Publ. 48, 61-71 (2011)

6. Karpuz, B., Santra, S.S.: Oscillation theorems for second-order nonlinear delay differential equations of neutral type. Hacet. J. Math. Stat. 48(3), 633-643 (2019)

7. Pinelas, S., Santra, S.S.: Necessary and sufficient condition for oscillation of nonlinear neutral first-order differential equations with several delays. J. Fixed Point Theory Appl. 20(1), 27 (2018)

8. Wong, J.S.W.: Necessary and suffcient conditions for oscillation of second order neutral differential equations. J. Math. Anal. Appl. 252(1), 342-352 (2000)

9. Grace, S.R., Džurina, J., Jadlovska, I., Li, T.: An improved approach for studying oscillation of second-order neutral delay differential equations. J. Inequal. Appl. 2018, 193 (2018)

10. Agarwal, R.P., Bohner, M., Li, T., Zhang, C.: Oscillation of second order differential equations with a sublinear neutral term. Carpath. J. Math. 30, 1-6 (2014)

11. Abdalla, B., Abdeljawad, T.: On the oscillation of Caputo fractional differential equations with Mittag-Leffler nonsingular kernel. Chaos Solitons Fractals 127, 173-177 (2019)

12. Abdalla, B., Abodayeh, K., Abdeljawad, T., Alzabut, J.: New oscillation criteria for forced nonlinear fractional difference equations. Vietnam J. Math. 45, 609-618 (2017)

13. Abdalla, B., Abdeljawad, T.: On the oscillation of Hadamard fractional differential equations. Adv. Differ. Equ. 409, 1-12 (2018)

14. Abdalla, B., Alzabut, J., Abdeljawad, T.: On the oscillation of higher order fractional difference equations with mixed nonlinearities. Hacet. J. Math. Stat. 47(2), 207-217 (2018)

15. Baculikova, B., Dzurina, J.: Oscillation theorems for second order nonlinear neutral differential equations. Comput. Math. Appl. 62, 4472-4478 (2011)

16. Baculikova, B., Li, T., Dzurina, J.: Oscillation theorems for second order neutral differential equations. Electron. J. Qual. Theory Differ. Equ. 74, 1 (2011)

17. Bazighifan, O., Elabbasy, E.M.: Oscillation of higher-order differential equations with distributed delay. J. Inequal. Appl. 2019, 55 (2019)

18. Bazighifan, O., Dassios, I.: Riccati technique and asymptotic behavior of fourth-order advanced differential equations. Mathematics 8, 1-11 (2020)

19. Bazighifan, O., Ruggieri, M., Santra, S.S., Scapellato, A.: Qualitative properties of solutions of second-order neutral differential equations. Symmetry 12(9), 1-10 (2020)

20. Santra, S.S., Bazighifan, O., Ahmad, H., Chu, Y.-M.: Second-order differential equation: oscillation theorems and applications. Math. Probl. Eng. 2020, Article ID 8820066 (2020). https://doi.org/10.1155/2020/8820066

21. Santra, S.S., Dassios, I., Ghosh, T.: On the asymptotic behavior of a class of second-order non-linear neutral differential equations with multiple delays. Axioms 9, 134 (2020). https://doi.org/10.3390/axioms9040134

22. Karpuz, B., Santra, S.: New criteria for the oscillation and asymptotic behavior of second-order neutral differential equations with several delays. Turk. J. Math. 44, 1990-2003 (2020). https://doi.org/10.3906/mat-2006-103

23. Santra, S.S., Bazighifan, O., Ahmad, H., Yao, S.-W.: Second-order differential equation with multiple delays: oscillation theorems and applications. Complexity 2020, Article ID 8853745 (2020). https://doi.org/10.1155/2020/8853745

24. Santra, S.S., Ghosh, T., Baghifan, O.: Explicit criteria for the oscillation of second-order differential equations with several sub-linear neutral coefficients. Adv. Differ. Equ. 2020, 643 (2020). https://doi.org/10.1186/s13662-020-03101-1

25. Li, T., Rogovchenko, Y.V.: Oscillation theorems for second order nonlinear neutral delay differential eqquations. Abstr. Appl. Anal. 2014, Article ID 594190 (2014)

26. Qian, Y., Xu, R.: Some new oscillation criteria for higher order quasi-linear neutral delay differential equations. Differ. Equ. Appl. 3, 323-335 (2011) 
27. Pinelas, S., Santra, S.S.: Necessary and sufficient conditions for oscillation of nonlinear first order forced differential equations with several delays of neutral type. Analysis 39(3), 97-105 (2019)

28. Ragusa, M.A.: Elliptic boundary value problem in vanishing mean oscillation hypothesis. Comment. Math. Univ. Carol. 40(4), 651-663 (1999)

29. Ragusa, M.A., Tachikawa, A.: Regularity for minimizes for functional of double phase with variable exponents. Adv. Nonlinear Anal. 9, 710-728 (2020)

30. Santra, S.S.: Existence of positive solution and new oscillation criteria for nonlinear first order neutral delay differential equations. Differ. Equ. Appl. 8(1), 33-51 (2016)

31. Santra, S.S.: Oscillation analysis for nonlinear neutral differential equations of second order with several delays. Mathematica 59(82), 111-123 (2017)

32. Santra, S.S.: Oscillation analysis for nonlinear neutral differential equations of second order with several delays and forcing term. Mathematica 61(84), 63-78 (2019)

33. Santra, S.S.: Necessary and sufficient condition for oscillatory and asymptotic behavior of second-order functional differential equations. Kragujev. J. Math. 44(3), 459-473 (2020)

34. Santra, S.S., Dix, J.G.: Necessary and sufficient conditions for the oscillation of solutions to a second-order neutral differential equation with impulses. Nonlinear Stud. 27(2), 375-387 (2020)

35. Yang, Q., Xu, Z:: Oscillation criteria for second order quasi-linear neutral delay differential equations on time scales. Comput. Math. Appl. 62, 3682-3691 (2011)

36. Ye, L., Xu, Z.: Oscillation criteria for second order quasilinear neutral delay differential equations. Appl. Math. Comput. 207, 388-396 (2009)

\section{Submit your manuscript to a SpringerOpen ${ }^{\circ}$ journal and benefit from:}

- Convenient online submission

- Rigorous peer review

- Open access: articles freely available online

- High visibility within the field

- Retaining the copyright to your article

Submit your next manuscript at $\boldsymbol{~ s p r i n g e r o p e n . c o m ~}$ 